\title{
Baseline Predictors for Good Versus Poor Visual Outcomes in the Treatment of Neovascular Age-Related Macular Degeneration With Intravitreal Anti-VEGF Therapy
}

\author{
Bora Chae, ${ }^{1,2}$ Jesse J. Jung, ${ }^{1-4}$ Sarah Mrejen, ${ }^{2,3}$ Roberto Gallego-Pinazo, ${ }^{2,3,5}$ Nicolas A. Yannuzzi, ${ }^{6}$ \\ Samir N. Patel, ${ }^{6}$ Christine Y. Chen, $, 2,7,8$ Marcela Marsiglia, ${ }^{2-4}$ Sucharita Boddu, ${ }^{1}$ \\ and K. Bailey Freund ${ }^{1-4}$
}

\author{
${ }^{1}$ Department of Ophthalmology, New York University School of Medicine, New York, New York, United States \\ ${ }^{2}$ Vitreous Retina Macula Consultants of New York, New York, New York, United States \\ ${ }^{3}$ LuEsther T. Mertz Retinal Research Center, Manhattan Eye, Ear and Throat Hospital, New York, New York, United States \\ ${ }^{4}$ Edward S. Harkness Eye Institute, Columbia University College of Physicians and Surgeons, New York, New York, United States \\ ${ }^{5}$ Department of Ophthalmology, University and Polytechnic Hospital La Fe, Valencia, Spain \\ ${ }^{6}$ Weill Cornell Medical College, New York, New York, United States \\ ${ }^{7}$ Department of Surgery, Monash University, Melbourne, Australia \\ ${ }^{8}$ Centre for Eye Research Australia, University of Melbourne, East Melbourne, Australia
}

Correspondence: K. Bailey Freund, Vitreous, Retina, Macula Consultants of New York, 460 Park Avenue, Fifth Floor, New York, NY 10022, USA; kbfnyf@aol.com.

Submitted: January 19, 2015 Accepted: June 22, 2015

Citation: Chae B, Jung JJ, Mrejen S, et al. Baseline predictors for good versus poor visual outcomes in the treatment of neovascular age-related macular degeneration with intravitreal antiVEGF therapy. Invest Ophthalmol Vis Sci. 2015;56:5040-5047.

DOI:10.1167/iovs.15-16494
Purpose. To examine the baseline factors associated with good (20/60 or better) versus poor (20/200 or worse) visual outcomes in eyes with treatment-naïe neovascular age-related macular degeneration (AMD) receiving intravitreal antivascular endothelial growth factor (VEGF) on a treat-and-extend regimen (TER).

MeTHoDs. An observational, retrospective series of patients managed with a TER, identified as having either good or poor visual outcomes, was examined. A multivariate regression analysis of baseline characteristics identified factors associated with good and poor vision at 2,3 , and 4 years. Neovascular subtypes were identified using fluorescein angiography (FA) alone and the anatomic classification system with FA and optical coherence tomography (OCT).

REsults. One hundred thirty-eight patients (154 eyes) fit the inclusion criteria at 2 years, 106 patients ( 113 eyes) at 3 years, and 72 patients ( 74 eyes) at 4 years. In the multivariate analysis, type 1 lesions, according to anatomic classification, had better vision at 24 months (95\% CI: [3.1, 82.7], $P=0.01$ ), 36 months (95\% CI: [1.97, 24.17], $P=0.003)$, and 48 months (95\% CI: $[2.01,65.47], P=0.006)$. Clopidogrel use was associated with poor vision at 24 months (95\% CI: $[0.03,0.68], P=0.013)$. Vision at 3 months was the best predictor of vision at year $4(\beta=$ $-4.277, P=0.002)$.

Conclusions. Eyes with neovascular AMD managed with a TER of anti-VEGF therapy having type 1 neovascularization at baseline were more likely to maintain good vision over 4 years, whereas clopidogrel use predicted poor vision at 2 years. Vision at 3 months was the best predictor for favorable long-term vision.

Keywords: age-related macular degeneration, treat-and-extend regimen, anatomical classification system, anti-VEGF, choroidal neovascularization

\begin{abstract}
A d actering dvanced age-related macular degeneration (AMD) is characterized by the presence of geographic atrophy (GA) or choroidal neovascularization (CNV), leading to irreversible loss of central vision. ${ }^{1}$ The identification of vascular endothelial growth factor (VEGF) as a major mediator of angiogenesis led to the development of intravitreal pharmacologic agents targeting VEGF and revolutionized the management of neovascular AMD. These drugs, which include bevacizumab (Avastin; Genentech, San Francisco, CA, USA), ranibizumab (Lucentis; Genentech), and aflibercept (Eylea; Regeneron, Tarrytown, NY, USA), are now the mainstay treatment for neovascular AMD after several clinical trials demonstrated prevention of vision loss and the possibility for vision improvement with regular use of these agents over 2 years. ${ }^{2-6}$ When comparing the clinical efficacy of bevacizumab versus ranibizumab in the treatment of neovas-
\end{abstract}

cular AMD, the Comparison of Age-related Macular Degeneration Treatments Trials (CATT) demonstrated equal efficacy between the two drugs in improving visual acuity of treated participants. ${ }^{7}$ Detailed analysis of this landmark trial revealed a variance in treatment response between patients. While more than two-thirds of participants in CATT had the same or improved visual acuity at 2 years compared to baseline visual acuity, up to $9 \%$ demonstrated loss of 15 or more letters. ${ }^{7}$ When analyzing the cohort of cases that exemplified sustained visual acuity loss in the CATT, factors contributing to the vision loss seen were the development of foveal scar, pigmentary abnormalities, or GA. ${ }^{8}$

Recent studies have shown that final visual outcomes vary between treated patients depending on several baseline factors. For instance, Freund and colleagues 9 showed that clinical 
response to anti-VEGF treatment is largely dependent on the neovascular lesion subtype. Using the anatomic classification of neovascularization (NV), accomplished by using both fluorescein angiography (FA) and optical coherence tomography (OCT) as described by Jung and associates, ${ }^{10}$ Freund and associates showed that type 1 neovascularization had the best long-term visual outcome in comparison to other lesion subtypes that were treated with a treat-and-extend regimen (TER). ${ }^{9}$ In comparison, poor visual outcome at 1 year after treatment with ranibizumab or bevacizumab has been linked to older age, better baseline visual acuity, large CNV lesion area, predominantly or minimally classic lesion subtypes, absence of retinal angiomatous proliferation (RAP) lesion, presence of GA, greater total foveal thickness, and retinal pigment epithelium (RPE) elevation at baseline on OCT. ${ }^{11}$

Identifying predictive factors for good and poor visual outcomes with anti-VEGF therapy allows for more accurate prediction of prognosis based on the patient's baseline characteristics. The main purpose of this study was to further explore the baseline predictors for long-term good and poor visual outcomes in the treatment of neovascular AMD with anti-VEGF therapy utilizing a TER at 4 years based on demographic and clinical factors including the classification of neovascular lesions determined by imaging with FA alone, as well as anatomically using both FA and OCT.

\section{Methods}

This study design was approved by the Western Institutional Review Board (Olympia, WA, USA). It complied with the Health Insurance Portability and Accountability Act of 1996 and followed the tenets of the Declaration of Helsinki.

\section{Data Collection}

In this retrospective cohort study, data collection was performed on the same cohort as previously described by Jung and associates ${ }^{10}$ using the charts and imaging data of 210 patients who underwent initiation of intravitreal anti-VEGF treatment with ranibizumab $(0.5 \mathrm{mg} / 0.05 \mathrm{~mL})$, bevacizumab $(1.25 \mathrm{mg} / 0.05 \mathrm{~mL})$, or aflibercept $(2.0 \mathrm{mg} / 0.05 \mathrm{~mL})$ with a TER by a single physician (KBF) for treatment-naïe neovascular AMD in one or both eyes between January 2006 and January 2013 at two offices of a private practice, Vitreous Retina Macula Consultants of New York.

The inclusion and exclusion criteria for the initial cohort were previously described by Jung and associates. ${ }^{10}$ All participants were older than 50 years with new-onset, treatment-naïve neovascular AMD in the absence of permanent foveal structural damage as evidenced by clinical examination and FA. For this specific cohort analysis, patients must have also had at least 24 months of follow-up, and only participants with a best-corrected visual acuity of $20 / 60$ or better or 20/200 or worse at each of 24,36 , and 48 months follow-up were included in the analysis. Eyes in the study also must have had OCT imaging (time-domain or spectral-domain) performed at the time of initial diagnosis.

Demographic information was collected for each patient, including age at first injection; sex; race; family history of AMD; smoking status (current, former, never); history of hypertension and diabetes; history of statin, aspirin, clopidogrel, and/or warfarin use; and history of glaucoma.

Participants received injections under a TER in which eyes were given topical proparacaine hydrochloride $(0.5 \%)$ and topical $5 \%$ povidone-iodine solution prior to injection. All injections were performed 3.5 to $4.0 \mathrm{~mm}$ posterior to the limbus with either a 30- or 32-gauge needle for ranibizumab
$(0.5 \mathrm{mg} / 0.05 \mathrm{~mL})$ and aflibercept $(2.0 \mathrm{mg} / 0.05 \mathrm{~mL})$ or a $31-$ gauge needle for bevacizumab $(1.25 \mathrm{mg} / 0.05 \mathrm{~mL})$. For the purpose of this study, no distinction was made between the anti-VEGF agents utilized. As previously described, all eyes received three monthly (4-6 weeks apart) loading doses followed by maintenance therapy at intervals increasing by 1 to 2 weeks per visit if visual acuity was stable, OCT displayed no intra- and subretinal fluid, and hemorrhage had resolved.9,12 The presence of pigment epithelial detachment (PED) on examination did not influence treatment intervals, and the treatment interval was extended to a maximum of 10 weeks unless signs of active neovascular disease occurred. The number of anti-VEGF injections an eye received, along with best-corrected visual acuity (Snellen) and intraocular pressure at each time point, was recorded from the chart review. The time points evaluated were baseline, 3 months, 6 months, and then every 6 months until the last visit at 4 years of follow-up. Visual acuity was converted from Snellen chart to logMAR for statistical analysis.

\section{Image Grading}

Color and FA images were obtained using a Topcon TRC 50IX fundus camera (Topcon, Tokyo, Japan). Optical coherence tomography imaging of all patients was performed with timedomain OCT (Stratus; Carl Zeiss Meditec, Inc., Dublin, CA, USA) or spectral-domain OCT (Spectralis, Heidelberg Engineering, Heidelberg, Germany; or 3-D OCT-2000, Topcon, Tokyo, Japan). Optical coherence tomography information was necessary for the anatomic grading of lesion subtypes. ${ }^{13}$ Standard methods of image acquisition were employed for all imaging modalities.

The classification of neovascular lesions, including type 1 (sub-RPE), type 2 (subretinal), type 3 (intraretinal), and type 4 (mixed), was made independently by two experienced retina specialists (SM and RG-P) who evaluated the presenting color photographs, FA, and OCT. Each neovascular lesion was classified according to the FA alone and with the anatomic classification system as previously detailed by Jung and associates. ${ }^{10}$ Baseline lesion and images were reviewed based on the Digital Angiographic Reading Center Reader's Manual. A third supervising grader (KBF) evaluated the lesion type in the presence of significant discrepancies. Readers also graded the lesion location and overall size. Fluorescein angiography was used to measure the greatest linear diameter $(\mathrm{mm})$ and the total area of CNV lesion $\left(\mathrm{mm}^{2}\right)$. Measurements were performed only on fundus camera images. The total area of CNV lesions was defined as the area of CNV leakage plus any contiguous areas of thick hemorrhage, blocked fluorescence, or serous PED that could be obscuring the boundaries of the CNV. The lesion location was defined as foveal (subfoveal or juxtafoveal) or extrafoveal as determined according to the MPS terminology. ${ }^{14}$ Specifically, foveal location was defined as the most posterior border of the lesion, including blood or blocked fluorescence involving the geometrical center of the fovea as observed on FA.

\section{Statistical Analysis}

All statistical analysis was done using SPSS Version 21 (SPSS, Inc., Chicago, IL, USA). Eyes with visual acuity of $20 / 60$ or better were compared with eyes with $20 / 200$ or worse at 24 , 36 , and 48 months. An additional analysis was performed on eyes with a visual acuity of $20 / 40$ or better and compared to eyes with $20 / 200$ or worse at 24,36 , and 48 months. Baseline categorical traits were compared using a two-sided Fisher's exact test. Baseline continuous variables were compared using a two-sample, two-tailed $t$-test assuming either equal variance 
TABLE 1. Patient Demographic and Baseline Clinical Characteristics

\begin{tabular}{|c|c|c|c|}
\hline & $\begin{array}{l}24 \mathrm{mo} \\
n=138\end{array}$ & $\begin{array}{l}36 \mathrm{mo} \\
n=106\end{array}$ & $\begin{array}{l}48 \mathrm{mo} \\
n=72\end{array}$ \\
\hline $\begin{array}{l}\text { Mean age at first injection } \\
\text { (SD), y }\end{array}$ & $80.5(8.5)$ & $80(8.6)$ & $79.7(8.3)$ \\
\hline Bilaterality, $n$ (\%) & $16(12)$ & $7(7)$ & $2(3)$ \\
\hline Female sex, $n(\%)$ & $88(64)$ & $72(68)$ & $45(63)$ \\
\hline Hypertension, $n$ (\%) & $84(61)$ & $65(61)$ & $45(64)$ \\
\hline Diabetes, $n(\%)$ & $18(13)$ & $9(8)$ & $11(15)$ \\
\hline Glaucoma, $n(\%)$ & $10(7)$ & $8(8)$ & $3(4)$ \\
\hline Smoking, $n(\%)$ & $39(28)$ & $23(22)$ & $16(22)$ \\
\hline Family history of AMD, $n$ (\%) & $28(20)$ & $21(22)$ & $9(13)$ \\
\hline Statin, $n(\%)$ & $47(34)$ & $30(28)$ & $24(33)$ \\
\hline Aspirin, $n(\%)$ & $49(36)$ & $37(35)$ & $27(38)$ \\
\hline Clopidogrel, $n$ (\%) & $10(7)$ & $4(4)$ & $3(4)$ \\
\hline Warfarin, $n(\%)$ & $7(5)$ & $6(6)$ & $5(7)$ \\
\hline $\begin{array}{l}\text { Average lesion area of } \mathrm{CNV} \\
\text { (SD), } \mathrm{mm}^{2}\end{array}$ & $7.1(6.6)$ & $6.8(6.2)$ & $6.8(6.2)$ \\
\hline $\begin{array}{l}\text { Average greatest linear } \\
\text { diameter (SD), mm }\end{array}$ & $3.3(1.5)$ & $3.2(1.3)$ & $3.2(1.3)$ \\
\hline $\begin{array}{l}\text { Average number of injections } \\
\text { per y (SD) }\end{array}$ & $8(1.6)$ & $8(1.6)$ & $8(1.4)$ \\
\hline $\begin{array}{l}\text { Mean interval between } \\
\text { injections (SD), wk }\end{array}$ & $6.6(1.5)$ & $6.6(1.5)$ & $6.7(1.4)$ \\
\hline
\end{tabular}

or unequal variance as determined by Levene's test of homogeneity of variance.

Specific clinical characteristics of interest were further assessed for a potential independent effect on visual acuity through a multivariate regression model. Based on the univariate analyses and limitations from the sample size, certain variables were not included for further evaluation. The multivariate regression models were separated into visual acuity (VA) clinical characteristics (VA baseline and VA at various time points) and non-VA clinical characteristics. Variables that resulted in a $P$ value $<0.2$ from the univariate analyses were evaluated using a binominal logistic regression at 24, 36, and 48 months. Variance inflation factors were calculated to control for collinearity among the predictors and to avoid confounding. Interaction terms were considered one at a time. All $P$ values were two-sided with statistical significance at the $0.05 \propto$ level. Ninety-five percent confidence intervals (95\% CI) for adjusted odds ratios (OR) were constructed to assess the precision of the obtained estimates.

\section{Results}

A total of 210 patients who were newly diagnosed with treatment-naïve neovascular AMD in at least one eye and treated with anti-VEGF therapy between January 2006 and

TABLE 2. Breakdown of Antivascular Endothelial Growth Factor Therapy Over 2, 3, and 4 Years

\begin{tabular}{lccc}
\hline & y 2 & y 3 & y 4 \\
\hline Bevacizumab only (\%) & $6(4 \%)$ & $3(3 \%)$ & $1(1 \%)$ \\
Ranibizumab only (\%) & $80(52 \%)$ & $58(51 \%)$ & $32(43 \%)$ \\
Aflibercept only & 0 & 0 & 0 \\
B+R (\%) & $26(17 \%)$ & $22(19 \%)$ & $19(26 \%)$ \\
B+A & 0 & 0 & 0 \\
R+A (\%) & $31(20 \%)$ & $19(17 \%)$ & $16(22 \%)$ \\
All 3 (\%) & $11(7 \%)$ & $11(10 \%)$ & $6(8 \%)$ \\
\hline
\end{tabular}

A, aflibercept; B, bevacizumab; R, ranibizumab.
TABLE 3. Frequency of Anatomic Classification, CNV Localization, and FA Classification Types

\begin{tabular}{|c|c|c|c|}
\hline & $\begin{array}{c}24 \text { mo, } \\
\text { Number (\%), } \\
n=154\end{array}$ & $\begin{array}{c}36 \text { mo, } \\
\text { Number (\%), } \\
n=113\end{array}$ & $\begin{array}{c}48 \mathrm{mo}, \\
\text { Number (\%), } \\
n=74\end{array}$ \\
\hline \multicolumn{4}{|c|}{ Anatomic classification } \\
\hline 1 & $61(39.6)$ & $42(37.2)$ & $29(39.2)$ \\
\hline 2 & $14(9.09)$ & $13(11.5)$ & $7(9.46)$ \\
\hline 3 & $47(30.5)$ & $32(28.3)$ & $20(27)$ \\
\hline Mixed NV & $32(20.8)$ & $26(23)$ & $18(24.3)$ \\
\hline \multicolumn{4}{|c|}{ CNV localization } \\
\hline Foveal & $103(66.9)$ & $76(67.3)$ & $54(73)$ \\
\hline Juxta- & $27(17.5)$ & $20(17.7)$ & $10(13.5)$ \\
\hline Extra- & $24(15.6)$ & $17(15)$ & $10(13.5)$ \\
\hline \multicolumn{4}{|c|}{ FA classification } \\
\hline Occult & $77(50)$ & $53(46.9)$ & $31(41.9)$ \\
\hline Classic & $20(13)$ & $19(16.8)$ & $14(18.9)$ \\
\hline RAP & $40(26)$ & $28(24.8)$ & $21(28.4)$ \\
\hline Mixed NV & $17(11)$ & $13(11.5)$ & $8(10.8)$ \\
\hline
\end{tabular}

January 2013 were analyzed. The numbers of patients who met the eligibility criteria for visual acuity of $20 / 60$ or better or visual acuity of 20/200 or worse were 138 (154 eyes) at 2 years, 106 (113 eyes) at 3 years, and $72(74$ eyes) at 4 years.

Table 1 summarizes patient demographics and baseline clinical characteristics of patients analyzed at 24, 36, and 48 months in the study. The breakdown of anti-VEGF treatments for each eye is included in Table 2. The frequency of NV lesion types based on the anatomic classification and FA classification is illustrated in Table 3 at 24, 36, and 48 months. Table 3 also shows the frequency of $\mathrm{CNV}$ localization in the three cohorts. The third supervising grader (KBF) was required to arbitrate the CNV classification seven times (5\%) with interpretation of OCT images and five times (3\%) with FA at year 2. At year 3, arbitrations occurred four times (4\%) with OCT images and four times (4\%) with FA. At year 4, arbitrations occurred one time (1\%) with OCT images and two times (3\%) with FA.

Table 4 demonstrates baseline characteristics associated with good (eyes with $20 / 60$ or better) or poor visual outcome (eyes with $20 / 200$ or worse). In this univariate analysis, we found that the anatomic classification of $\mathrm{CNV}$, hypertension, clopidogrel use, injection mean interval, injections per year, and the number of injections at 12 and 24 months were associated with good or poor visual outcome at 24 months. At 36 months, baseline factors associated with good or poor visual outcome were anatomic classification, mean injection interval, injections per year, and number of injections at 36 months. At 48 months, anatomic classification, FA classification, mean injection interval, injections per year, number of injections at 48 months, and clopidogrel use were associated with good or poor visual outcome.

The multivariate analysis for baseline clinical characteristics at 24,36 , and 48 months is shown in Table 5. Type 1 lesions by the anatomic classification system were significant predictors of good visual outcome of $20 / 60$ or better at 24 months $(95 \%$ CI: [3.1, 82.7], $P=0.01), 36$ months (95\% CI: [1.97, 24.17], $P=$ 0.003 ), and 48 months (95\% CI: [2.01, 65.47], $P=0.006)$. Eyes with type 3 lesions at 24 months (95\% CI: [2.06, 45.07], $P=$ $0.004)$ and 36 months (95\% CI: [1.12, 11.32], $P=0.032$ ) were significant predictors of good visual outcome, but this was not seen at 48 months (95\% CI: $[0.44,6.55], P=0.437)$. The number of injections was not a significant predictor of visual acuity in the multivariate analysis at 24,36 , and 48 months of follow-up. Clopidogrel use in patients was a significant 
TABLe 4. Univariate Analysis of Baseline Characteristics and Long-Term Visual Acuity at 24, 36, and 48 Months

\begin{tabular}{|c|c|c|c|}
\hline & $\begin{array}{c}\text { Visual Acuity at } 24 \text { mo, } \\
\text { No. of Eyes }\end{array}$ & $\begin{array}{c}\text { Visual Acuity at } 36 \mathrm{mo}, \\
\text { No. of Eyes }\end{array}$ & $\begin{array}{c}\text { Visual Acuity at } 48 \mathrm{mo} \text {, } \\
\text { No. of Eyes }\end{array}$ \\
\hline Eyes with $20 / 60$ or better & 117 & 81 & 52 \\
\hline Eyes with $20 / 200$ or worse & 37 & 32 & 22 \\
\hline \multirow[t]{2}{*}{ Total eyes } & 154 & 113 & 74 \\
\hline & $P$ Values & $P$ Values & $P$ Values \\
\hline Age at first injection & 0.126 & 0.262 & 0.090 \\
\hline Anatomic classification & 0.001 & 0.001 & 0.004 \\
\hline Aspirin & 0.545 & 0.141 & 0.215 \\
\hline Bilaterality & 0.790 & 0.643 & 0.586 \\
\hline Choroidal atrophy & 0.461 & 0.145 & 0.364 \\
\hline CNV localization & 0.377 & 0.936 & 1.000 \\
\hline Coumadin & 0.233 & 0.349 & 0.630 \\
\hline Diabetes mellitus & 0.410 & 0.728 & 0.216 \\
\hline Family history of AMD & 0.433 & 0.790 & 0.801 \\
\hline Fluorescein angiography classification & 0.263 & 0.174 & 0.048 \\
\hline Glaucoma & 0.534 & 0.263 & 1.000 \\
\hline Greatest linear diameter & 0.376 & 0.623 & 0.285 \\
\hline Hypertension & 0.036 & 0.138 & 0.223 \\
\hline Injection mean interval & 0.000 & 0.010 & 0.004 \\
\hline Injections per $\mathrm{y}$ & 0.000 & 0.003 & 0.006 \\
\hline No. injections at $3 \mathrm{mo}$ & 0.265 & 0.777 & 0.841 \\
\hline No. injections at $6 \mathrm{mo}$ & 0.194 & 0.291 & 0.114 \\
\hline No. injections at $12 \mathrm{mo}$ & 0.021 & 0.321 & 0.197 \\
\hline No. injections at $24 \mathrm{mo}$ & 0.006 & 0.083 & 0.102 \\
\hline No. injections at $36 \mathrm{mo}$ & - & 0.017 & 0.052 \\
\hline No. injections at $48 \mathrm{mo}$ & - & - & 0.012 \\
\hline Overall lesion area & 0.107 & 0.671 & 0.446 \\
\hline Clopidogrel & 0.014 & 0.318 & 0.024 \\
\hline Sex & 0.568 & 0.270 & 0.487 \\
\hline Smoking history & 0.115 & 0.441 & 0.442 \\
\hline Statins & 0.840 & 0.532 & 0.399 \\
\hline Visual acuity at presentation & 0.000 & 0.000 & 0.000 \\
\hline Visual acuity at $3 \mathrm{mo}$ & 0.000 & 0.000 & 0.000 \\
\hline Visual acuity at $6 \mathrm{mo}$ & 0.000 & 0.000 & 0.000 \\
\hline Visual acuity at $12 \mathrm{mo}$ & 0.000 & 0.000 & 0.000 \\
\hline Visual acuity at $24 \mathrm{mo}$ & - & 0.000 & 0.000 \\
\hline Visual acuity at $36 \mathrm{mo}$ & - & - & 0.000 \\
\hline
\end{tabular}

${ }^{*}$ Univariate clinical significance, $P$ value $<0.20$.

predictor of poor visual outcome at 24 months (95\% CI: [0.03, 0.68 ], $P=0.013$ ), but this significance was not found at 36 months (95\% CI: $[0.03,3.18], P=0.335)$. Clopidogrel use was not included in the multivariate analysis at 48 months due to the lack of statistical power. Fluorescein angiography-determined lesion type and CNV localization were not significant predictors of visual acuity at 24 months of follow-up.

Table 6 shows the multivariate analysis for the visual acuity characteristics at each time point. At each time point, visual acuities at baseline, at 3 months, and at each previous year of follow-up were included as clinical parameters. Visual acuity at 12 months was found to be a strong predictor of vision at year $2(\beta=-6.577, P<0.001)$. Visual acuity at 24 months was a statistically significant predictor of vision at year $3(\beta=$ $-10.403, P=0.001)$. Visual acuity at 3 months was the best predictor of long-term visual acuity at year $4(\beta=-4.277, P=$ 0.002).

The number of patients who met criteria for visual acuity of $20 / 40$ or better or visual acuity of $20 / 200$ or worse was 108 ( 115 eyes) at 2 years, 84 ( 87 eyes) at 3 years, and 61 (61 eyes) at 4 years. In an additional multivariate analysis, type 1 lesions by the anatomic classification system were significant predictors of good visual outcome of $20 / 40$ or better at 24 months (95\% CI: [5.12, 813.08], $P=0.001), 36$ months (95\% CI: [1.84,
25.89], $P=0.004)$, and 48 months (95\% CI: [1.60, 53.88], $P=$ 0.01). Eyes with type 3 lesions at 24 months (95\% CI: [2.12, 216.62], $P=0.009$ ) were significant predictors of good visual outcome of $20 / 40$ or better. Clopidogrel use was a marginally significant independent predictor of poor vision at year $2(95 \%$ CI: $[0.05,1.03], P=0.055)$. Visual acuity at year 1 was a statistically significant predictor of vision at year $2(\beta=$ $-15.854, P=0.03)$ and at year $3(\beta=-14.903, P=0.005)$. Visual acuity at 3 months was the best predictor of visual acuity at year $4(\beta=-4.277, P=0.003)$.

\section{DisCussion}

In this study, we report the baseline factors that predict either good (20/60 or better) or poor (20/200 or worse) visual acuity at years 2, 3, and 4 in eyes on a TER of anti-VEGF therapy for treatment-naïve AMD. Previous studies have retrospectively analyzed cohorts from randomized clinical trials who received treatment at multiple clinical centers with varied treatment strategies during their follow-up period. For instance, the CATT study group analyzed a cohort of 61 patients who demonstrated sustained visual acuity loss of 15 letters or more after being treated monthly or pro re nata (PRN) for 2 years and found that 
TABLE 5. Multivariate Analysis of Baseline Nonvisual Acuity Clinical Parameters Predictive of Long-Term Visual Acuity

\begin{tabular}{|c|c|c|c|c|c|c|}
\hline \multirow[b]{2}{*}{ Clinical Characteristic } & \multicolumn{2}{|l|}{ y 2} & \multicolumn{2}{|c|}{ y 3} & \multicolumn{2}{|l|}{ y 4} \\
\hline & OR $(95 \% \mathrm{CI})$ & $P$ Value* & OR $(95 \% \mathrm{CI})$ & $\boldsymbol{P}$ Value* & OR $(95 \% \mathrm{CI})$ & $P$ Value* \\
\hline Anatomic classification $\dagger$ & & 0.04 & & 0.06 & & 0.032 \\
\hline 1 & $16.01(3.1-82.7)$ & 0.01 & $6.90(1.97-24.17)$ & 0.003 & $11.46(2.01-65.47)$ & 0.006 \\
\hline 2 & $0.85(0.17-4.34)$ & 0.848 & $0.90(0.23-3.53)$ & 0.884 & $0.77(0.12-4.82)$ & 0.782 \\
\hline 3 & $9.64(2.06-45.07)$ & 0.004 & $3.56(1.12-11.32)$ & 0.032 & $1.71(0.44-6.55)$ & 0.437 \\
\hline Injection no. $\neq$ & $1.14(0.98-1.32)$ & 0.084 & $1.07(0.97-1.19)$ & 0.172 & $1.10(0.99-1.22)$ & 0.078 \\
\hline Clopidogrel & $0.15(0.03-0.68)$ & 0.013 & $0.33(0.03-3.18)$ & 0.335 & & \\
\hline FA classification $₫$ & & 0.273 & & & & \\
\hline Occult & $0.18(0.03-1.13)$ & 0.067 & & & & \\
\hline Classic & $0.81(0.15-4.21)$ & 0.80 & & & & \\
\hline RAP & $0.27(0.04-1.64)$ & 0.15 & & & & \\
\hline CNV localization $\|$ & & 0.697 & & & & \\
\hline Foveal & $0.91(0.25-3.37)$ & 0.889 & & & & \\
\hline Juxtafoveal & $1.57(0.30-8.25)$ & 0.597 & & & & \\
\hline
\end{tabular}

No additional clinical parameters apart from those displayed at the respective time points were included in the regression. Bold values are statistically significant.

* $P$ values are two-sided with statistical significance evaluated at the $0.05 \alpha$ level.

$\dagger$ Reference standard for anatomic classification was type 4 (mixed lesions).

‡ Injection number is computed relative to each given time frame (i.e., injection number at 2 years).

$\S$ Reference standard for FA classification was mixed.

|| Reference standard for CNV localization was extrafoveal.

the presence of baseline nonfoveal GA, larger lesion size, and bevacizumab treatment were independent predictive factors for sustained vision loss at 2 years. ${ }^{8}$

Analyses of the correlations between baseline characteristics and visual outcomes in eyes treated for more than 2 years are limited. There are two major prospective studies that investigated the long-term effects of anti-VEGF treatment beyond 2 years: the Open-Label Extension Trial of Ranibizumab for Choroidal Neovascularization Secondary to Age-Related Macular Degeneration (HORIZON) and the Seven-Year Outcomes in Ranibizumab-Treated Patients in ANCHOR, MARINA, and HORIZON multicenter cohort study (SEVEN-UP) trials. ${ }^{15,16}$ The HORIZON study was a 2-year extension from the initial 2year MARINA, ANCHOR, and the RhuFab V2 Ocular Treatment Combining the Use of Visudyne to Evaluate Safety (FOCUS) trials. In this study, patients were switched from monthly injections to PRN regimens in which treatment was given based on the discretion of the treating physician with patient examined every 3 months or more frequently per examiner's discretion. Based on the analysis of 100 patients who were previously treated with monthly intravitreal ranibizumab in the initial studies, good visual outcome (equal to or greater than 15-letter gains) at month 48 was associated with younger age and lower initial baseline visual acuity. ${ }^{15}$ The SEVEN-UP study evaluated a cohort of patients originally enrolled in MARINA, ANCHOR, and HORIZON at multiple treatment centers who were then treated on a PRN basis out to a 7-year follow-up. In this study, only 65 patients were analyzed, and the authors found that total lesion area (leaking CNV components, staining scar, subretinal hemorrhage, blocked fluorescence, and serous pigment epithelial detachment) plus nonlesion components (RPE disturbance and atrophy) affected long-term visual acuity at 7 years. ${ }^{16}$

In comparison, we analyzed a cohort managed by a single physician (KBF) over a 4-year period with a consistent TER. We analyzed the baseline predictors for either good (20/60 or better) or poor (20/200 or worse) visual outcomes in response to anti-VEGF treatment at 2,3 , and 4 years of follow-up. This cohort is more representative of the current real-world population in which most patients with neovascular AMD in the United States are being treated with anti-VEGF agents on a TER. According to the 2014 American Society of Retina Specialists Preferences and Trends (ASRS PAT) annual survey, approximately $77.9 \%$ of U.S. retina specialists report using the TER to manage their patients with neovascular AMD. ${ }^{17}$ The TER regimen helps achieve a more individualized treatment approach to AMD while alleviating some of the large burden on patients and physicians by decreasing the frequency of office visits and ancillary testing needed compared to alternative dosing regimens. ${ }^{12,18-20}$ Several short-term studies evaluating outcomes with a TER have demonstrated favorable visual outcomes that appear similar to those with fixed-monthly dosing regimens. ${ }^{12,21}$ Most recently, the Lucentis Compared to Avastin study (LUCAS), the first prospective randomized

TABLE 6. Multivariate Analysis of Visual Acuity Parameters Predictive of Long-Term Visual Acuity

\begin{tabular}{|c|c|c|c|c|c|c|}
\hline \multirow[b]{2}{*}{ Visual Acuity Parameters } & \multicolumn{2}{|c|}{ у 2} & \multicolumn{2}{|c|}{ y 3} & \multicolumn{2}{|c|}{ y 4} \\
\hline & B & $P$ Value & B & $P$ Value & B & $P$ Value \\
\hline VA baseline & -2.136 & 0.203 & 0.338 & 0.854 & -0.503 & 0.665 \\
\hline VA 3 mo & -3.514 & 0.071 & -1.863 & 0.349 & -4.277 & 0.002 \\
\hline VA $12 \mathrm{mo}$ & -6.577 & $<0.001$ & & & & \\
\hline VA $24 \mathrm{mo}$ & & & -10.403 & 0.001 & & \\
\hline
\end{tabular}

B values are $\beta$ values of the regression. $P$ values are two-sided with statistical significance evaluated at the $0.05 \alpha$ level. No additional clinical parameters apart from those displayed at the respective time points were included in the regression. Bold values are statistically significant. 
multicenter trial comparing the efficacy of ranibizumab and bevacizumab for the treatment of neovascular AMD using a TER protocol, found that the two drugs had equivalent effect on vision at 1 year. ${ }^{22}$ Visual acuity results obtained at 1 year were comparable to those of other clinical trials with monthly treatment. $^{22}$ Investigation of long-term outcomes using the TER beyond 12 months is limited. However, a recent study by Rayess and associates ${ }^{23}$ utilizing a TER showed improvement in visual acuity from baseline that was maintained up to 3 years of follow-up in an analysis of 59 eyes. To our knowledge, the present study provides an analysis of baseline predictive characteristics for long-term vision on the largest cohort of patients with the longest follow-up to date who were managed with a TER by a single physician.

While the HORIZON study documented younger age and lower baseline VA as baseline predictors for improved vision at 48 months, our analysis did not reveal an association between these factors and long-term visual outcome. We found that age $(P=0.09)$ and baseline visual acuity $(\beta=-0.503, P=0.665)$ did not have a significant association with good or poor long-term vision. The HORIZON study also found that a smaller area of lesion and leakage (in the controlled randomized group only) was associated with visual gain at 24 months. However, our study did not find overall lesion area $(P=0.107)$ to be a predictor of good visual outcome at 24 months. The SEVEN-UP study also noted that the presence of leakage or total area of CNV leakage did not significantly influence visual acuity at 7 years. While this study examined somewhat different baseline CNV lesion characteristics, we similarly found that CNV lesion size (area of CNV leakage plus any contiguous areas of thick hemorrhage, blocked fluorescence, or serous PED) was not associated with long-term visual acuity at 4 years $(P=0.446)$.

In our analysis, favorable visual acuity was associated with type 1 (sub-RPE) lesions based on the anatomic classification at $24(P=0.01), 36(P=0.003)$, and 48 months $(P=0.006)$. Similar findings were reported by Freund and colleagues. ${ }^{9}$ Type 1 lesions present in the sub-RPE layer are characterized by neovascularization that may form as a compensatory mechanism to provide nutrients and oxygen to the outer retina. ${ }^{24}$ Grossniklaus and associates ${ }^{24}$ hypothesized that the essential nutrients that continually replenish the RPE and outer retina may be present in the chronic subretinal fluid, possibly explaining why type 1 lesions appear linked to favorable long-term outcomes. Further evidence supporting this hypothesis was shown in an analysis by $\mathrm{Xu}$ and associates, ${ }^{25}$ who found that eyes with type 1 neovascularization treated with intravitreal anti-VEGF therapy developed less GA compared to all other lesion types. Grunwald and associates ${ }^{26}$ retrospec- $^{-}$ tively analyzed the 2-year follow-up data from the CATT group and found that the presence of RAP was also associated with a higher risk of development of GA, making it a strong predictor of poor visual outcome, while occult lesions or sub-RPE lesions did not have an overall worse vision.

Our study failed to show a clinically significant association between good visual acuity and occult CNV diagnosed using FA alone. With the addition of OCT to assess neovascular phenotype, the anatomic classification system may be a more clinically relevant method to define lesion subtypes. ${ }^{10}$

In terms of poor prognostic factors, clopidogrel use in patients was found to be a statistically significant predictor of poor visual outcome at 24 months $(P=0.013)$. At 24 months, more patients ( 154 eyes total) were included in our analysis compared to 36 (113 eyes total) and 48 months ( 74 eyes total), allowing for more statistical power at year 2 in comparison to the analysis performed at subsequent follow-up points.

Our finding between clopidogrel and poor visual outcome in neovascular AMD might relate to the association between neovascular AMD and cardiovascular disease. Studies have documented the role of common inflammatory markers in the pathogenesis of both AMD and cardiovascular disease. Polymorphism in the complement factor $\mathrm{H}$ gene and other genes responsible for the regulation of the complement pathway has been shown to be strongly associated with neovascular AMD. ${ }^{27,28}$ Moreover, the age-related maculopathy susceptibility 2 gene (ARMS2, rs10490924) is strongly associated with exudative $\mathrm{AMD}^{29}$ and has been linked to elevated CRP. ${ }^{30,31}$ Both the complement pathway and higher levels of CRP have been implicated in cardiovascular disease. ${ }^{32}$ In a recent, large population-based study of AMD patients, Vassilev and associates ${ }^{33}$ reported a small but significant increased risk of AMD in patients with cardiovascular comorbidities including myocardial infarction, heart failure, or hyperlipidemia. ${ }^{33}$ Similarly, Wang and associates ${ }^{34}$ found that early AMD was independently associated with coronary stenosis.

Clopidogrel is used to prevent death and ischemic complications in patients with prior history of myocardial infarction, stroke, or peripheral arterial disease; patients who have undergone percutaneous coronary intervention; and patients with unstable angina. ${ }^{35-37}$ More recently, the use of clopidogrel has been extended to patients with the most severe manifestation of coronary artery disease: myocardial infarction associated with ST-segment elevation. ${ }^{38}$ The use of clopidogrel in patients is indicative of patients with prior or current significant cardiovascular disease considering the possible link between AMD and underlying systemic vascular disease, a poor prognostic factor may exist for AMD patients with significant ischemic heart disease taking clopidogrel.

Interestingly, we found that visual acuity at 3 months was a better predictive factor of good long-term visual acuity at 4 years $(P=0.002)$ as opposed to baseline visual acuity. In contrast to our findings, several studies have reported baseline VA to be a statistically significant predictor of final VA outcome. ${ }^{11,39,40}$ In comparison, these studies had only 1 or 2 years of follow-up. Rasmussen and associates, ${ }^{41}$ who followed treated patients up to 4 years, found that while higher baseline visual acuity was a predictor of good visual outcome, vision after three injections at 3 months was also associated with better outcome at 4 years. In this same study, visual acuity at 3 months was a stronger predictor of vision at 4 years than baseline vision $\left(P<0.0001\right.$ versus $P=0.005$, respectively) ${ }^{41}$ Our findings also demonstrate that treatment response seen after the initial three anti-VEGF injections may be the best predictive factor for favorable long-term good visual acuity. Not surprisingly, the most recent visual acuity from the year prior to analysis at 24 and 36 months was also a significant predictor of good vision. The most recent visual acuity may also demonstrate patients who are continually responsive to therapy and therefore predict maintenance of good long-term visual results. Further analysis on this subset of patients and their anatomic characteristics may allow identification of more clinical predictors linked to good outcomes.

Findings similar to those mentioned above were obtained with utilization of a more stringent criterion for good visual outcome (20/40 or better). Type 1 lesions by the anatomical classification were again found to be a strong predictor of good visual outcome at 24, 36, and 48 months. Visual acuity at 3 months was still the best predictor of long-term visual outcome at 48 months. However, vision at 24 months was no longer a predictor of vision at 36 months, and clopidogrel was only a marginally significant independent predictor of poor vision at 24 months $(P=0.055)$. With a $20 / 60$ criteria for "good" vision, more patients were included in the analysis, which allowed for more statistical power to find a statistical significance between the two factors

Limitations of this study include its retrospective nature and the use of different OCT devices including the time-domain 
and spectral-domain OCT at baseline, which may have affected the initial grading of the lesion; but we previously showed that there is overall good agreement when classifying CNV lesions with either time-domain or spectral-domain OCT. ${ }^{10}$ Two independent graders were utilized to classify lesion types, producing some inherent biases that could have affected the results. However, any disagreements were arbitrated by a third supervising grader (KBF).

In addition, different anti-VEGF agents were utilized in the TER of our cohort of patients, including the most recent federal drug administration (FDA)-approved anti-VEGF agent, aflibercept (Regeneron), which may have affected the long-term visual outcomes. While these inherent limitations exist, our results are highly reflective of the real-world clinical setting using a TER performed by a single physician and may allow clinicians to predict the long-term clinical response to multiple forms of antiVEGF therapy in newly diagnosed neovascular AMD.

Furthermore, because a small percentage of patients were lost to follow-up for various reasons including death, transfer of care due to relocation, switch to a PRN strategy, or missed or delayed appointments, not all patients studied at 24 months were included in 36- and 48-month data analysis; similarly, not all patients examined at 36 months were included at 48 months. For this reason, we acknowledge this as a weakness with respect to the conclusions of our study regarding the long-term visual outcome.

In conclusion, in eyes with neovascular AMD treated with intravitreal anti-VEGF therapy on a TER, the presence of type 1 neovascularization at baseline appears to be a strong predictor of good visual outcomes at 2, 3, and 4 years. The use of clopidogrel appears to predict worse visual outcomes at 2 years. Visual acuity at 3 months was the best predictor for favorable long-term visual outcomes at 4 years. Identifying these baseline predictive factors for good and poor visual outcomes is clinically relevant, as this information allows clinicians to better categorize patients and counsel them with respect to their long-term visual prognosis.

\section{Acknowledgments}

Presented at the annual meeting of the Association for Research in Vision and Ophthalmology, Orlando, Florida, United States, May 2014

Supported by a research grant from the LuEsther T. Mertz Retinal Research Center, Manhattan Eye, Ear and Throat Hospital, and The Macula Foundation, Inc. The authors alone are responsible for the content and writing of the paper.

Disclosure: B. Chae, None; J.J. Jung, None; S. Mrejen, None; R. Gallego-Pinazo, Bayer HealthCare (F, C, R), Carl Zeiss Meditec (C, R), Novartis (F, C, R), Heidelberg Engineering (F), Thea (F), Sensimed (F); N.A. Yannuzzi, None; S.N. Patel, None; C.Y. Chen, None; M. Marsiglia, None; S. Boddu, None; K.B. Freund, Regeneron (C, R), Genentech (C, R), Bayer HealthCare (C, R), ThromboGenics (C), Heidelberg Engineering (C, R)

\section{References}

1. Coleman HR, Chan CC, Ferris FL III, Chew EY. Age-related macular degeneration. Lancet. 2008;372:1835-1845.

2. Avery RL, Pieramici DJ, Rabena MD, et al. Intravitreal bevacizumab (Avastin) for neovascular age-related macular degeneration. Ophthalmology. 2006;113:363-372.

3. Rosenfeld PJ, Brown DM, Heier JS, et al. Ranibizumab for neovascular age-related macular degeneration. $N$ Engl J Med. 2006;355:1419-1431.

4. Martin DF, Maguire MG, Ying GS, Grunwald JE, Fine SL, Jaffe GL. Ranibizumab and bevacizumab for neovascular age-related macular degeneration. $N$ Engl J Med. 2011;364:1897-1908.
5. Brown DM, Kaser PK, Michels M, et al. Ranibizumab versus verteporfin for neovascular age-related macular degeneration. N Engl J Med. 2006;355:1432-1444.

6. Heier JS, Brown DM, Chong V, et al. Intravitreal aflibercept (VEGF trap-eye) in wet age-related macular degeneration. Ophthalmology. 2012;119:2537-2548.

7. Comparison of Age-related Macular Degeneration Treatments Trials (CATT) Research Group, Martin DF, Maguire MG, et al. Ranibizumab and bevacizumab for treatment of neovascular age-related macular degeneration: two-year results. Ophthalmology. 2012;119:1388-1398.

8. Ying G, Kim BJ, Maguire MG, et al. Sustained visual acuity loss in the comparison of age-related macular degeneration treatments trials. JAMA Ophthalmol. 2014;132:915-921.

9. Freund KB, Korobelnik JF, Devenyi R, et al. Treat-and-extend regimens with anti-VEGF agents in retinal disease: a literature review and consensus recommendations. Retina. In press.

10. Jung JJ, Chen CY, Mrejen S, et al. The incidence of neovascular subtypes in newly diagnosed neovascular age-related macular degeneration. Am J Ophthalmol. 2014;158:769-779.

11. Ying G, Huang J, Maguire MG, et al. Baseline predictors for one year visual outcomes with ranibizumab or bevacizumab for neovascular age-related macular degeneration. Ophthalmology. 2013;120:122-129.

12. Engelbert M, Zweifel SA, Freund KB. "Treat and extend" dosing of intravitreal antivascular endothelial growth factor therapy for type 3 neovascularization/retinal angiomatous proliferation. Retina. 2009;29:1424-1431.

13. Freund KB, Zweifel SA, Engelbert M. Do we need a new classification for choroidal neovascularization in age-related macular degeneration? Retina. 2010;30:1333-1349.

14. Macular Photocoagulation Study Group. Subfoveal neovascular lesions in age-related macular degeneration. Guidelines for evaluation and treatment in the macular photocoagulation study. Arch Ophthalmol. 1991;109:1242-1257.

15. Singer MA, Awh CC, Sadda S, et al. HORIZON: an open-label extension trial of ranibizumab for choroidal neovascularization secondary to age-related macular degeneration. Ophthalmology. 2012;119:1175-1183.

16. Rofagha S, Bhisitkul RB, Boyer DS, Sadda SR, Zhang K. Sevenyear outcomes in ranibizumab-treated patients in ANCHOR, MARINA, and HORIZON: a multicenter cohort study (SEVENUP). Ophthalmology. 2013;120:2292-2299.

17. Stone TW, ed. ASRS 2014 Preferences and Trends Membership Survey. Chicago, IL: American Society of Retina Specialists; 2014.

18. Gupta OP, Shienbaum G, Patel AH, et al. A treat and extend regimen using ranibizumab for neovascular age-related macular degeneration: clinical and economic impact. Ophthalmology. 2010;117:2134-2140.

19. Shienbaum G, Gupta O, Fecarotta C, et al. Bevacizumab for neovascular age-related macular degeneration using a treatand-extend regimen: clinical and economic impact. Am J Ophthalmol. 2012;153:468-473.

20. Engelbert M, Zweifel SA, Freund KB. Long-term follow-up for type 1 (subretinal pigment epithelium) neovascularization using a modified "treat and extend" dosing regimen of intravitreal antivascular endothelial growth factor therapy. Retina. 2010;30:1368-1375.

21. Abedi F, Wickremasinghe S, Islam AFM, Inglis KM, Guymer RH. Anti-VEGF treatment in neovascular age-related macular degeneration: a treat-and-extend protocol over 2 years. Retina. 2014;34:1531-1538.

22. Berg K, Pedersen TR, Sandvik L, Bragadóttir R. Comparison of ranibizumab and bevacizumab for neovascular age-related macular degeneration according to LUCAS treat-and-extend protocol. Ophthalmology. 2015;122:146-152. 
23. Rayess N, Houston SK III, Gupta OP, Ho AC, Regillo CD. Treatment outcomes after 3 years in neovascular age-related macular degeneration using a treat-and-extend regimen. $A m J$ Ophthalmol. 2015;159:3-8.

24. Grossniklaus HE, Green WR. Choroidal neovascularization. Am J Opbthalmol. 2004;137:496-503.

25. Xu L, Mrejen S, Jung JJ, et al. Geographic atrophy in patients receiving anti-vascular endothelial growth factor for neovascular age-related macular degeneration. Retina. 2015;35:176186.

26. Grunwald JE, Daniel E, Huang J, et al.; CATT Research Group. Risk of geographic atrophy in the comparison of age-related macular degeneration treatments trials. Ophthalmology. 2014; 121:150-161.

27. Edwards AO, Ritter R III, Abel KJ, et al. Complement factor $\mathrm{H}$ polymorphism and age-related macular degeneration. Science. 2005;308:421-424.

28. Hughes $\mathrm{AE}$, Orr $\mathrm{N}$, Esfandiary $\mathrm{H}$, et al. A common $\mathrm{CFH}$ haplotype, with deletion of CFHR1 and CFHR3, is associated with lower risk of age-related macular degeneration. Nat Genet. 2006;38:1173-1177.

29. Keilhauer CN, Fritsche LG, Guthoff R, Haubitz I, Weber BH. Age-related macular degeneration and coronary heart disease: evaluation of genetic and environmental associations. Eur J Med Genet. 2013;56:72-79.

30. Seddon JM, Gensler G, Milton RC, et al. Association between C-reactive protein and age-related macular degeneration. JAMA. 2004;291:704-710.

31. Yasuma TR, Nakamura M, Nishiguchi KM, et al. Elevated Creactive protein levels and ARMS2/HTRA1 gene variants in subjects without age-related macular degeneration. Mol Vis. 2010;16:2923-2930.

32. Cao JJ, Arnold AM, Manolio TA, et al. Association of carotid artery intima-media thickness, plaques, and C-reactive protein with future cardiovascular disease and all-cause mortality: the Cardiovascular Health Study. Circulation. 2007;116:32-38.
33. Vassilev ZP, Ruigómez A, Soriano-Gabarró M, et al. Diabetes, cardiovascular morbidity, and risk of age-related macular degeneration in a primary care population. Invest Ophthalmol Vis Sci. 2015;10;56:1585-1592.

34. Wang SB, Mitchell P, Chiha J, et al. Severity of coronary artery disease is independently associated with the frequency of early age-related macular degeneration. $\mathrm{Br} J$ Ophthalmol. 2015;99:365-370

35. CAPRIE Steering Committee. A randomised, blinded, trial of clopidogrel versus aspirin in patients at risk of ischaemic events (CAPRIE). Lancet. 1996;348:1329-1339.

36. Steinhubl SR, Berger PB, Mann JT III, et al. Early and sustained dual oral antiplatelet therapy following percutaneous coronary intervention: a randomized controlled trial. JAMA. 2002;288: 2411-2420.

37. The Clopidogrel in Unstable Angina to Prevent Recurrent Ischemic Events Trial Investigators. Effects of clopidogrel in addition to aspirin in patients with acute coronary syndromes without ST-segment elevation. $N$ Engl J Med. 2001;345:494502.

38. Sabatine MS, Cannon CP, Gibson CM, et al.; for the CLARITYTIMI 28 Investigators. Addition of clopidogrel to aspirin and fibrinolytic therapy for myocardial infarction with ST-segment elevation. $N$ Engl J Med. 2005;352:1179-1189.

39. Boyer DS, Antoszyk AN, Awh CC, et al.; MARINA Study Group. Subgroup analysis of the MARINA study of ranibizumab in neovascular age-related macular degeneration. Ophthalmology. 2007; 114:246-252.

40. Kaiser PK, Brown DM, Zhang K, et al. Ranibizumab for predominantly classic neovascular age-related macular degeneration: subgroup analysis of first-year ANCHOR results. $\mathrm{Am} \mathrm{J}$ Ophthalmol. 2007;144:850-857.

41. Rasmussen A, Block SB, Fuchs J, et al. A 4-year longitudinal study of 555 patients treated with ranibizumab for neovascular age-related macular degeneration. Ophthalmology. 2013;120: 2630-2636. 


\section{University Library}

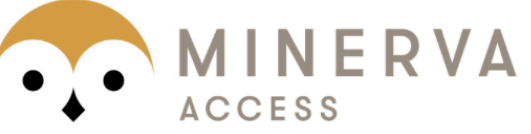

A gateway to Melbourne's research publications

Minerva Access is the Institutional Repository of The University of Melbourne

\section{Author/s:}

Chae, B;Jung, JJ;Mrejen, S;Gallego-Pinazo, R;Yannuzzi, NA;Patel, SN;Chen, CY;Marsiglia, M;Boddu, S;Freund, KB

Title:

Baseline Predictors for Good Versus Poor Visual Outcomes in the Treatment of Neovascular Age-Related Macular Degeneration With Intravitreal Anti-VEGF Therapy

Date:

2015-08-01

Citation:

Chae, B., Jung, J. J., Mrejen, S., Gallego-Pinazo, R., Yannuzzi, N. A., Patel, S. N., Chen, C. Y., Marsiglia, M., Boddu, S. \& Freund, K. B. (2015). Baseline Predictors for Good Versus Poor Visual Outcomes in the Treatment of Neovascular Age-Related Macular Degeneration With Intravitreal Anti-VEGF Therapy. INVESTIGATIVE OPHTHALMOLOGY \& VISUAL SCIENCE, 56 (9), pp.5040-5047. https://doi.org/10.1167/iovs.15-16494.

Persistent Link:

http://hdl.handle.net/11343/118275 